\title{
8. Relative Magnitude of the Preliminary and the Principal Portions of Earthquake Motions.
}

\author{
By Takeo Matsuzawa. \\ Seismological Institute, Tokyo Imperial University.
}

(Rec. Oct. 30, 1925. Comm. by Akitune Imamura, M.I.A., Dec. 12;1925.)

Since the pendulum observations of earthquake motions have been introduced in seismometry, the existence of preliminary tremors has been brought to light, and in the earlier days of the history of seismology, several tentative explanations ${ }^{1)}$ were given of their existence. The following is another contribution to the subject, based upon certain facts which have not yet been considered by the earlier workers.

Whatever the mechanism of earthquake occurrence may be, several kinds of elastic waves are excited by a common cause or causes, and this fact must be taken into account in the study of all earthquakes. The diversities of the transmitting mediums (i.e. rocks) may, of course, modify the nature of these waves in different ways, but we know that the nature of an earthquake motion depends first of all and not a little on the very mechanism of the occurrence of each earthquake. The chief object of this paper is to examine the effect of the mode of excitation on the character of the resulting earthquake motions.

In this preliminary work, the medium is assumed to be jsotropic, homogeneous, and infinitely extended. The well known equations of STok Es, ${ }^{2)}$ modified by Love, ${ }^{3)}$ may, therefore, be most conveniently used

(1) J.A. Ewing, Trans. Seism. Soc. Japan, 3 (1881) 121.

J. Milne, " " " " 8 (1885) 182.

C.G. KNott, ," " " " , 12 (1888) 115.

H. NAGaOKA, Publ. Imp. Earthq. Invest. Commit. No. 4. (1900).

S. Kusakabe, " " " " " No. 14. (1903).

A. Imamura, " " $\quad, \quad " \quad$, (in Japanese)No.99.(1925)18.

(2) G.G. Stokes, Mathem. and Phys. Papers Vol. II, On the Dynamical Theory of Diffraction (1849).

(3) A.E.H. Love, London Mathem. Soc. Proc. (Ser. 2), 1 (1904). 
for the purpose. When a force $\chi(t)$ acts at a point in an infinite elastic solid in the direction of $\mathrm{X}$ axis, the displacements are given by

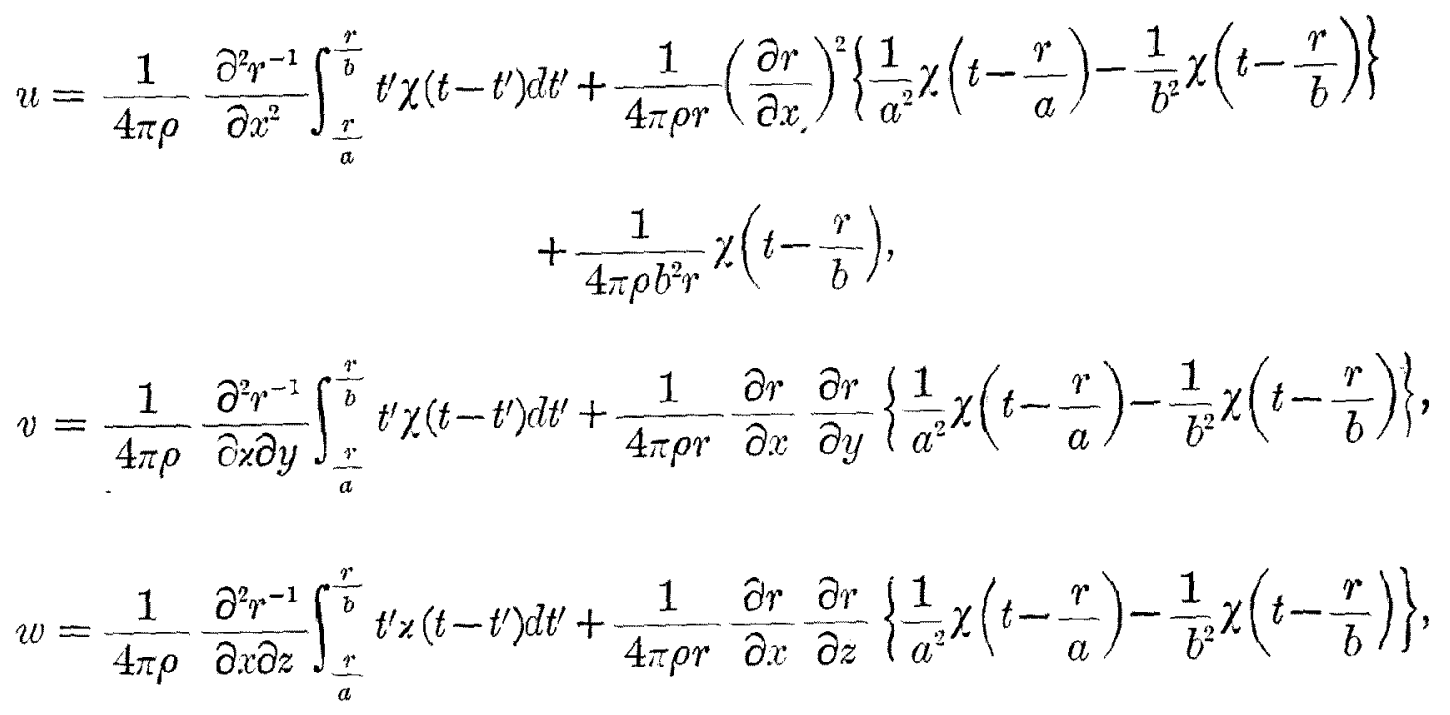
where $\rho$ is the density of the medium,

$a$ the velocity of the longitudinal wave, $b, ", \quad$, transverse wave,

$r$ the distance between the source and the point of observation.

Combining this singlet in different ways, expressions for different modes of excitations may be obtained. Several cases have been examined, assuming in each case, the force $\chi(t)$ to be of an oscillatory character, that is, $\mathrm{e}^{\mathrm{ipt}}$.

The chief results are as follows :-

(a) In some special cases, the direction of motion of the dilatational wave may not necessarily lie in the direction of propagation. Similar remarks may also be made in the case of the distorsional wave.

(b) In ordinary cases, the ratio of amplitude of the dilatational wave to that of the distorsional wave is given by $\frac{\lambda+2 \mu}{\lambda}$, where $\lambda$ and $\mu$ are LAMé's constants. That is, the distorsional component is ordinarily greater than the dilatational component, as usually observed. However, in some particular cases, the magnitude of the dilatational wave may be greater than that of the distorsional wave.

(c) Roughly speaking, the distorsional wave component is far greater 
No. 1.] Relative Magnitude of the Preliminary and the Principal Portion etc.

than the dilatational wave component, when the cause of excitation is of a sliding, rotational, or distorsional character. When it is of a compressional character or the like, the dilatational component may be greater. These relations are capable of a rational explanation.

(d) The amplitude generally diminishes as the reciprocal of the distance from the origin. This does not, however, hold in all cases.

(e) Velocities of propagation of wave forms are dependent on the mechanism of excitation, direction of propagation, periods of oscillation, and the distance from the origin. But the velocities always tend to approach those of the plane waves (i.e. $a$ and $b$ ), as the distance increases.

Actual cases may be quoted which compare favourably with the theory above outlined.*

* After this paper was written, the author came across a paper by K. SudA (Umi to Sora, 5 (1925), No. 3. Japanese), in which similar problems are treated in a similar way, at least in principle. But he assumes that the displacements in an elastic medium caused by a singlet are polarized, an assumption which has rendered his solutions unduly simple. 